\title{
Analysis of bodily fluids using Vibrational Spectroscopy: A direct comparison of Raman scattering and Infrared absorption techniques for the case of glucose in blood serum
}

Drishya Rajan Parachalil ${ }^{\mathrm{a}, \mathrm{b}^{*}}$, Clément Bruno ${ }^{\mathrm{c}, \mathrm{d} ; \mathrm{e}}$, Franck Bonnier ${ }^{\mathrm{c}}$, Hélène Blasco ${ }^{\mathrm{d}, \mathrm{e}}$, Igor Chourpa $^{\mathrm{c}}$, Matthew J. Baker ${ }^{\mathrm{f}, \mathrm{g}}$, Jennifer McIntyre ${ }^{\mathrm{a}}$, and Hugh J. Byrne ${ }^{\mathrm{a}}$

${ }^{a}$ FOCAS Research Institute, Technological University Dublin, Kevin Street, Dublin 8, Ireland

${ }^{\mathrm{b}}$ School of Physics and Optometric \& Clinical Sciences, Technological University Dublin, Kevin Street, Dublin 8, Ireland

c Université de Tours, UFR sciences pharmaceutiques, EA 6295 Nanomédicaments et Nanosondes, 31 avenue Monge, 37200 Tours, France.

${ }^{\mathrm{d}}$ CHRU de Tours, Laboratoire de Biochimie et Biologie Moléculaire, Tours, France.

${ }^{\text {e } U n i v e r s i t e ́ ~ d e ~ T o u r s, ~ i B r a i n, ~ U M R ~ I N S E R M ~ U 1253, ~ 37032, ~ F r a n c e . ~}$

e WestCHEM, Department of Pure \& Applied Chemistry, Technology and Innovation Centre, University of Strathclyde, Glasgow, G1 1RD, UK

${ }^{f}$ ClinSpec Diagnostics Ltd (ClinSpec Dx), Level 7, Technology and Innovation Centre, 99 George Street, Glasgow, G1, 1RD, UK

*Corresponding Author: drishyarajan.parachalil@ mydit.ie

\begin{abstract}
Analysis of biomarkers present in the blood stream can potentially deliver crucial information on patient health and indicate the presence of numerous pathologies. The potential of vibrational spectroscopic analysis of human serum for diagnostic purposes has been widely investigated and, in recent times, infrared absorption spectroscopy, coupled with ultra-filtration and multivariate analysis techniques, has attracted increasing attention, both clinical and commercial. However, such methods commonly employ a drying step, which may hinder the clinical work flow and thus hamper their clinical deployment. As an alternative, this study
\end{abstract}


explores the use of Raman spectroscopy, similarly coupled with ultra-filtration and multivariate analysis techniques, to quantitatively monitor diagnostically relevant changes of glucose in liquid serum samples, and compares the results with similar analysis protocols using infrared spectroscopy of dried samples. The analysis protocols to detect the imbalances in glucose using Raman spectroscopy are first demonstrated for aqueous solutions and spiked serum samples. As in the case of infrared absorption studies, centrifugal filtration is utilised to deplete abundant analytes and to reveal the spectral features of Low Molecular Weight Fraction analytes in order to improve spectral sensitivity and detection limits. Improved Root Mean Square Error of Cross Validation (RMSECV) was observed for Raman prediction models, whereas slightly higher $\mathrm{R}^{2}$ values were reported for infrared absorption prediction models. Summarising, it is demonstrated that the Raman analysis protocol can yield accuracies which are comparable with those reported using infrared absorption based measurements of dried serum, without the need for additional drying steps.

\section{Keywords}

Raman Spectroscopy; Glucose; Background correction; Extended Multiplicative Signal Correction (EMSC); Centrifugal filtration

\section{Introduction}

Human bodily fluids (e.g. blood serum/ plasma, urine, saliva, tears and cerebrospinal fluid) are considered to be a rich reservoir of clinical biomarkers and are an interesting alternative to cells and tissues in terms of disease diagnosis and prognosis, owing to advantages such as minimal invasiveness, low cost, and rapid sample collection and processing (1-4). The biochemical composition of human serum can provide crucial information on patient health and indicate the presence of numerous pathologies, as it encompasses a vast range of proteins and biochemical products accumulated while perfusing various organs (5). Moreover, alterations in the 
biochemical composition of the serum/plasma could reflect changes of physiological states due to disease, enabling early disease diagnosis and treatment (6-8). For example, it is reported that carcinoembryonic antigens, CA 15-3 and CA 27.29, can be considered as serum biomarkers for breast cancer (9), KL-40 and MMP-9 have been found to be potential serum biomarkers for high grade gliomas (10), prostate specific antigen was found in higher levels in the serum of patients with prostate cancer $(11,12)$, and carcinoembryonic antigen has been reported to be serum biomarker for colorectal cancer (13). In recent decades, extensive studies have explored the detection of serum biomarkers for various diseases, and quite naturally the reference tools used are the conventional, complex analytical techniques such as chromatography, electrophoresis or mass spectroscopy (14-18). More recently, the field of serum proteomics has exploded in the literature, as this emerging field has gained world-wide attention due to its potential to reveal important information regarding the pathogenesis or progression of diseases (19-22).

Analysis of serum biomarkers based on protein content is inherently challenging, because of the low concentrations and the vast variety and dynamic range of protein abundance. The complex nature of the serum poses as a huge problem for the detection of small molecule biomarkers (2,23). Human serum contains more than 10,000 different proteins, with an overall concentration ranging from $60-80 \mathrm{mg} / \mathrm{mL}$. Furthermore, circulating species present in the serum, such as metabolites, peptides, sugars, and lipids, add to its complexity. Conventional proteomic methods struggle to handle large dynamic range of abundances of its constituent components (17). The characteristics of serum are usually dominated by the high molecular weight fraction (HMWF) of proteins, which includes albumin (57-71\%) and globulin (8-26\%) and masks the features of low molecular weight analytes which are present in trace amounts $(<5 \%)$ (24). Chromatographic fractionation can significantly enhance the sensitivity and specificity of the data recorded, and coupling with spectroscopic analysis techniques such as 
IR absorption in hybrid techniques such as Liquid Chromatography (LC-IR) or Gas Chromatography (GC-IR) (25) can improve the performance further. Such chromatographic techniques, although extensively used in pharmaceutical, chemical or food science applications are time consuming and costly, however (26-28), and commercially available centrifugal filters have been employed to deplete the HMWF before analysis to facilitate analysis of the low molecular weight fraction (LMWF), including proteins and molecular biomarkers (29).

Vibrational spectroscopic techniques, both Raman and infrared absorption, have emerged over the past 20 years as increasingly routine analytical techniques for a wide range of applications, as they reveal specific biochemical information without the use of extrinsic labels. Although they are often considered complementary techniques (30) there are also specific considerations for each, for specific applications such as measurement of bodily fluids, as described by Bonnier et al. (31). They provide intrinsic vibrational signatures of the material of interest in a non-destructive fashion, and the potential for diagnostic applications has been well demonstrated, notably in human serum and plasma (7,32-34). However, although both Raman, Fourier-Transform Infrared (FTIR) and Attenuated Total Reflectance-FTIR (ATR-FTIR) spectroscopy have been widely explored to study bodily fluids over the last two decades, most of these studies have been carried out on air dried samples, in order to avoid the water contribution in the case of FTIR, and to increase the concentration of the analytes in the case of Raman (35-40). Two major limiting factors in the use of dried samples are the drying time (41) and also the so-called "coffee-ring" effect, or, specifically in terms of blood serum, the Vroman effect (42-44), whereby different analytes precipitate from solution at different rates, giving rise to variations in the spectral features due to chemical and physical inhomogeneity. Previous studies have clearly shown that the IR spectrum of dried (aggregates of) molecular species is not the same as that in solution (45). It has further been shown that, for dried deposits from solutions of varying concentrations of analyte, the linearity of the Beer-Lambert law, and 
therefore the quantitative nature of the measurement is compromised as the concentration of the analyte is increased (45). In the case of the "coffee ring effect", the thickness, and therefore the quantitative accuracy of the measurement is spatially inhomogeneous, and the technique is not ideally suited for quantitative measurements, such as those considered here. Notably, Spalding et al. have used a serum dilution technique to improve the reliability of the technique for quantitative measurement (46). The issues with inhomogeneity can potentially be overcome by micropipetting and sampling the whole drop, and there have been studies that show excellent specificity/sensitivity of classification of diseased state using dried samples $(40,47,48)$. Nevertheless, the requirement of a drying step adds considerably to the sampling time and complexity of the workflow $(41,49)$.

Protocols for monitoring changes in HMWF serum proteins in their native liquid form using Raman spectroscopy have recently been demonstrated, however, without the need for drying (50). Analysing in the native liquid state, the chemical composition is averaged out by molecular motion over the measurement time, greatly reducing the variability of the measurement. Bonnier et al. compared FTIR and Raman for measuring gelatin in solution and described protocols for isolation of LMWF from serum (31). This study indicated that Raman in the liquid form could deliver similar sensitivities to ATR-FTIR for measurement of LMWF species.

The aim of this study is to investigate the sensitivity and accuracy of Raman spectroscopy in the liquid state, coupled with centrifugal filtration, as a biochemical tool to detect clinically relevant changes in the biochemical composition of serum and compare to the ATR-FTIR technique, using the specific example of glucose. The study is specifically designed according to the ATR-FTIR study of glucose in serum by Bonnier et al., using identical parameters and protocols of ultra-filtration and multivariate regression analysis, such that a direct comparison 
of the two techniques can be made (29). The protocol is first demonstrated using aqueous solutions and human serum spiked with systematically varied concentrations of glucose, before exploring the sensitivity and accuracy of the technique in patient samples.

\section{Materials and Methods}

\subsection{Preparation of varying concentration of glucose in distilled water model}

D-glucose (G8769) was purchased from Sigma Aldrich, Ireland and 6 glucose solutions were prepared over the concentration range $100 \mathrm{mg} / \mathrm{dL}$ to $1000 \mathrm{mg} / \mathrm{dL}$. Amicon Ultra $0.5 \mathrm{~mL}$ centrifugal filter devices (Millipore- Merck, Germany), with 10kDa cut off point, were employed to concentrate and fractionate the serum samples. The centrifugation procedure previously reported by Bonnier et al. was followed (31). A further study published by Bonnier et al. reported $100 \%$ recovery of LMWF using $10 \mathrm{kDa}$, hence only $10 \mathrm{kDa}$ cut off filtration has been used in the present study (45). Pre-rinsing of the filter devices with $0.1 \mathrm{M} \mathrm{NaOH}$ prior to plasma analysis is essential to avoid glycerine interference in the analysis (32). The optimised washing and rinsing procedure includes spinning $0.5 \mathrm{~mL} 0.1 \mathrm{M} \mathrm{NaOH}$ at $14000 \times \mathrm{g}$ for 30 minutes followed by three rinses with distilled water by spinning $0.5 \mathrm{~mL}$ distilled water for 30 minutes at $14000 \times \mathrm{g}$. Every 30 minute wash and rinse must be followed by spinning the device in the inverted position at $1000 \times \mathrm{g}$ for 2 minutes, to remove the residual solution contained in the filter. After washing, $0.5 \mathrm{~mL}$ of glucose solution is transferred to the $10 \mathrm{kDa}$ filter and centrifuged at $14000 \times \mathrm{g}$ for 30 minutes. The solution that passes through the $10 \mathrm{kDa}$ filter is the filtrate, which contains mostly water and molecules smaller than 10kDa. The remainder of the serum, known as the concentrate, is collected by placing the filter device upside down and spinning for $1000 \times \mathrm{g}$ for 2 minutes. The resultant concentrate, $\sim 50 \mu \mathrm{L}$, contains molecules with molecular weight larger than $10 \mathrm{kDa}$, concentrated by a factor of $\sim 10$, and can be employed for study of the HMWF (50). All the filtrate solutions were analysed using Raman spectroscopy 
and five replicate measurements from different positions have been performed for each sample of $\sim 50 \mu \mathrm{L}$. In subsequent analysis, each patient is represented by all the spectra recorded from that patient, rather than the mean.

\subsection{Preparation of glucose spiked in serum model}

Sterile, human serum (H6194) and D-glucose (G8769) were purchased from Sigma Aldrich, Ireland, for the preparation of in vitro spiked models. The commercial human serum was spiked with glucose in the concentration range that matches of the study of Bonnier et al. (29). Since glucose is already present in normal human serum at concentrations of $70-110 \mathrm{mg} / \mathrm{dl}(51)$, the final concentration of the spiked samples covers the physiologically relevant ranges of normal (80-120mg/dL) (52), and hyperglycaemia (>120mg/dL). The centrifugal processing step described in Section 2.1 was performed on the spiked serum samples to obtain the filtrate. Note, in the analysis of Figure 3, the regression is performed over spiked, rather than total glucose concentration, consistent with the approach of Bonnier et al. (29).

\subsection{Glucose levels in patient serum samples}

Patient serum samples were donated by the University Hospital (CHU) Bretonneau de Tours (France) and the ethical procedures were followed. The blood samples were collected from the individuals as routine blood check-ups and $1 \mathrm{~mL}$ per patient was provided for spectroscopic analysis. A total of 25 patient samples were included in the present study. Samples were collected by personnel of $\mathrm{CHU}$, under standard clinical protocols and approved ethical procedures (Comité de Protection des Personnes, Tours Region Central Oest 1- PP/ANSMPHAO15-HB-METABOMU, registered internationally: ClinicalTrials.gov ID: NCT02670226). The samples were serologically profiled, for other purposes, and the anonymised, residual discard samples, along with their serological profiles were donated to the Université François-Rabelais de Tours, for further study. No further specific ethical approval 
or patient consent is required. Glucose concentrations were obtained by routine biochemical analysis using a COBAS analyser, following the in house guidelines for routine biochemical analysis. The principle of the test is based on the enzymatic reference method with hexokinase, which catalyses the phosphorylation of glucose to glucose-6-phosphate by ATP methods $(53,54)$. Subsequently, glucose-6-phosphate is oxidised by glucose-6-phosphate dehydrogenase, in the presence of NADP, to gluconate-6-phosphate. This reaction is specific, with no other carbohydrate being oxidised. The rate of NADPH formation during the reaction is directly proportional to the glucose concentration and is measured photometrically in the UV. Measured glucose levels in the patient samples are listed in Table 1, and can be seen to cover the range $55-435 \mathrm{mg} \mathrm{dL}^{-1}$. Note, that the distribution of glucose levels covers a broader range than that of the study of similar $(\mathrm{n}=15)$ patients by Bonnier et al. $\left(61-208 \mathrm{mg} \mathrm{dL}^{-1}\right)(29)$. 
Table 1: List of measured glucose levels in patient samples. Glucose blood levels are quoted in terms of the SI unit of $\mathrm{mmol} \mathrm{L}^{-1}$ as well as $\mathrm{mg} \mathrm{dL}^{-1}$, commonly used in serology literature and in the study of Bonnier et al.(29)

\begin{tabular}{|c|c|c|}
\hline \multirow[t]{2}{*}{ Sample number } & \multicolumn{2}{|c|}{ Glucose blood levels } \\
\hline & $\mathrm{mmol} \mathrm{L}^{-1}$ & $\mathrm{mg} \mathrm{dL}^{-1}$ \\
\hline 1 & 2.9 & 52.25 \\
\hline 2 & 3.1 & 55.85 \\
\hline 3 & 3.9 & 70.27 \\
\hline 4 & 3.9 & 70.27 \\
\hline 5 & 4.2 & 75.67 \\
\hline 6 & 4.2 & 75.67 \\
\hline 7 & 4.3 & 77.47 \\
\hline 8 & 4.5 & 81.08 \\
\hline 9 & 5.1 & 91.89 \\
\hline 10 & 5.1 & 91.89 \\
\hline 11 & 5.2 & 93.69 \\
\hline 12 & 5.7 & 102.70 \\
\hline 13 & 6 & 109.10 \\
\hline 14 & 6.1 & 109.90 \\
\hline 15 & 6.4 & 115.31 \\
\hline 16 & 6.4 & 115.31 \\
\hline 17 & 7.2 & 129.72 \\
\hline 18 & 8.8 & 158.55 \\
\hline 19 & 9.9 & 178.37 \\
\hline 20 & 11.5 & 207.20 \\
\hline 21 & 11.7 & 210.81 \\
\hline 22 & 13.4 & 241.44 \\
\hline 23 & 15.7 & 282.88 \\
\hline 24 & 15.8 & 284.68 \\
\hline 25 & 24.1 & 434.23 \\
\hline
\end{tabular}




\subsection{Data collection using Raman spectrophotometer}

The measurement conditions used for screening HMWF proteins in solution have recently been detailed (50). Raman spectra of all the liquid samples were recorded at stabilised room temperature $\left(18^{\circ} \mathrm{C}\right)$ using a Horiba Jobin-Yvon LabRam HR800 spectrometer with a 16-bit dynamic range Peltier cooled CCD detector. The spectrometer was coupled to an Olympus 1X71 inverted microscope and a x60 water immersion objective (LUMPlanF1, Olympus) was employed. The substrate used was a Lab-Tek plate (154534) with a $0.16-0.19 \mathrm{~mm}$ thick glass bottom, 1.0 borosilicate cover glass, and was purchased from Thermo Fischer Scientific, Ireland. Since the scattering efficiency is inversely proportional to the fourth power of the wavelength, it is always desirable to use a short wavelength Raman source. In the filtered serum, there are no molecular species which are resonant with 532nm, and therefore neither fluorescence nor photodamage are limiting factors.

\subsection{Data pre-processing and analysis}

The raw spectra were subjected to pre-processing techniques in Matlab before further analysis, to remove the background signal and reduce the noise. Smoothing of the raw data was done using the Savitzky-Golay method, with a polynomial order of 5 and window 13 and the Extended Multiplicative Signal Correction (EMSC) algorithm was applied to the smoothed spectra to remove the underlying water spectrum, whose $\mathrm{OH}$ bending feature at $1640 \mathrm{~cm}^{-1} \mathrm{can}$ interfere with the protein spectra, particularly at low concentrations (55).

The principle of EMSC for subtraction of a specific measureable background spectrum and the associated Matlab codes have previously been published by Kerr and Hennelly, 2016 (55), and their description has been adapted in the following. In the case of measurement in aqueous solution, the raw sample spectrum, S, consists of Raman spectrum of the analyte of interest, R, a baseline signal, $\mathrm{B}$, and the water signal, $\mathrm{W}$.

$\mathrm{S}=\mathrm{R}+\mathrm{B}+\mathrm{W}(55)$ 
The Raman spectrum of interest can be represented by a reference spectrum of the analyte of interest, $r$, and it can be assumed that $\mathrm{R}$ is the product of this reference spectrum and a certain scalar weight, $C_{r}$, which describes the concentration dependence $(56,57)$.

$\mathrm{R} \sim C_{r} \mathrm{x} \mathrm{r}(55)$

Similarly, a spectrum, w, is recorded from water directly in order to represent the spectral contribution of water in $\mathrm{W}$, as the product of pure water spectrum and a certain scalar weight.

$\mathrm{W}=C_{w} \mathrm{x} \mathrm{W}$

The baseline, $\mathrm{B}$, is now represented by a polynomial:

$\mathrm{B}_{N}=C_{0}+C_{1} \mathrm{X}+C_{2} \mathrm{X}+\ldots \ldots+C_{N} \mathrm{X}^{\mathrm{N}}$

where $N$ is the order of polynomial and $C_{\mathrm{m}}$ for $\mathrm{m}=0 \rightarrow \mathrm{N}$ represents various coefficients of polynomial (58). The EMSC algorithm is used to obtain estimates of the scalar values $C_{r}, C_{m}$ and $C_{w}$. These estimates are obtained from an optimal fit of the various vectors in Equation 5 . $\mathrm{S} \sim\left[C_{r} \times r\right]+\left[C_{w} \times w\right]+\left[\sum_{m=0}^{N} C_{m} X^{m}\right]$

The background corrected, concentration dependent analyte spectra, T, can be represented as:

$\mathrm{T}=\frac{\mathrm{S}-\left[C_{w} \times w\right]-\left[\sum_{m=0}^{N} C_{m X} m\right]}{C_{w}}$

Note, that division by $C_{w}$ has the effect of scaling the analyte spectra, assuming a constant water contribution to all sample spectra. The Raman spectrum of the stock glucose solution $(450 \mathrm{~g} / \mathrm{L})$ is used as the reference for EMSC, and polynomial of order 5 was used in all cases. The glucose reference spectrum and water spectrum used in EMSC were recorded using the 532nm laser line and Lab-Tek plate as substrate. The x60 objective brings the laser to a focus within the liquid, beyond the thin $(0.16 \mathrm{~mm}-0.19 \mathrm{~mm})$ glass substrate. No significant contribution from the glass to the recorded spectrum was apparent, and therefore no correction was required. The Rubberband method (59) was used to baseline correct the glucose reference spectrum after smoothing it using the Savitzky-Golay method. 


\subsubsection{Partial Least Squares Regression}

Partial Least Squares Regression (PLSR) was employed to establish a model that relates the variations of the spectral data to a series of concentrations. This regression model can be used to improve the limit of detection of Raman bio-sensing (60). Constructed based on the spectra of samples of known glucose content, either solutions of varying concentrations of glucose (in water or in commercial serum), or those of the patient serum, the model is then validated using a rigorous cross validation procedure which evaluates its performance in accurately predicting glucose concentrations. A 20 fold cross validation approach has been employed to validate the robustness of the method. This approach involves randomly dividing the set of observations into approximately equal size, $50 \%$ of the spectral data were randomly selected as test set, while the remaining $50 \%$ is used as the training set (61). In the current case, ( 5 x 25 spectra) were divided into two groups of 65 (test) and 60 (training) spectra. The cross-validation process is then repeated 20 times (the folds), with all observations are used for both training and testing, and each observation is used for testing exactly once. The results from the folds can then be averaged to produce a single estimation. The Root Mean Square Error of Cross Validation (RMSECV) is calculated from the 20 iterations to measure the performance of the model for the unknown cases within the calibration set. The correlation between the concentration and spectral intensity is given by the $\mathrm{R}^{2}$ value. The standard deviation was calculated to find the variation between each spectrum calculated from the same sample. The number of latent variables used for building the PLSR model is optimised by finding the value that is equivalent to the minimum of the RMSECV

\section{Results}


In this study, Raman spectra of the samples were recorded in the inverted geometry using a x60 water immersion objective with a 532nm laser and the Lab-Tek plate was used as the substrate. The $532 \mathrm{~nm}$ laser was chosen as it is compatible with the thin glass bottomed LabTek plate and provides a strong Raman signal of the sample with minimal background interference. This set-up was previously reported by Bonnier et al. (31) to yield better analysis of serum using Raman spectroscopy when the sample was analysed in the inverted geometry using a water immersion objective with a $785 \mathrm{~nm}$ laser and $\mathrm{CaF}_{2}$ substrate. The added advantage of this setup is that it provides high quality, consistent Raman spectra from sample volumes as low as $1 \mu \mathrm{L}$. The protocol using $532 \mathrm{~nm}$ was more recently further explored for analysis of HMWF serum proteins (50).

Figure 1 presents the spectra of the fingerprint region of the pure glucose solution recorded in the inverted geometry. The raw spectra of the glucose were baseline corrected using the rubberband method and smoothed using the Savitzky-Golay algorithm (polynomial 5, window 13). Example signature peaks of glucose (indicated by asterisks) appear at $\sim 450 \mathrm{~cm}^{-1}$, associated with an endocyclic $\delta(\mathrm{C}-\mathrm{C}-\mathrm{O})$ ring mode, $\delta(\mathrm{C} 1-\mathrm{H} 1)$ vibration at $911 \mathrm{~cm}^{-1}$, a peak at $1060 \mathrm{~cm}^{-1}$ due to $v(\mathrm{C} 1-\mathrm{OH})$ stretching, a relatively sharp peak at $1125 \mathrm{~cm}^{-1}$ which can be assigned to the $\delta(\mathrm{C}-\mathrm{O}-\mathrm{C})$ angle-bending mode and the sharp peaks at $1340 \mathrm{~cm}^{-1}$ and $1460 \mathrm{~cm}^{-1}$, related to the $\delta(\mathrm{C}-\mathrm{C}-\mathrm{H})$ vibration and pure $\mathrm{CH}_{2}$ group vibration, respectively(62). 


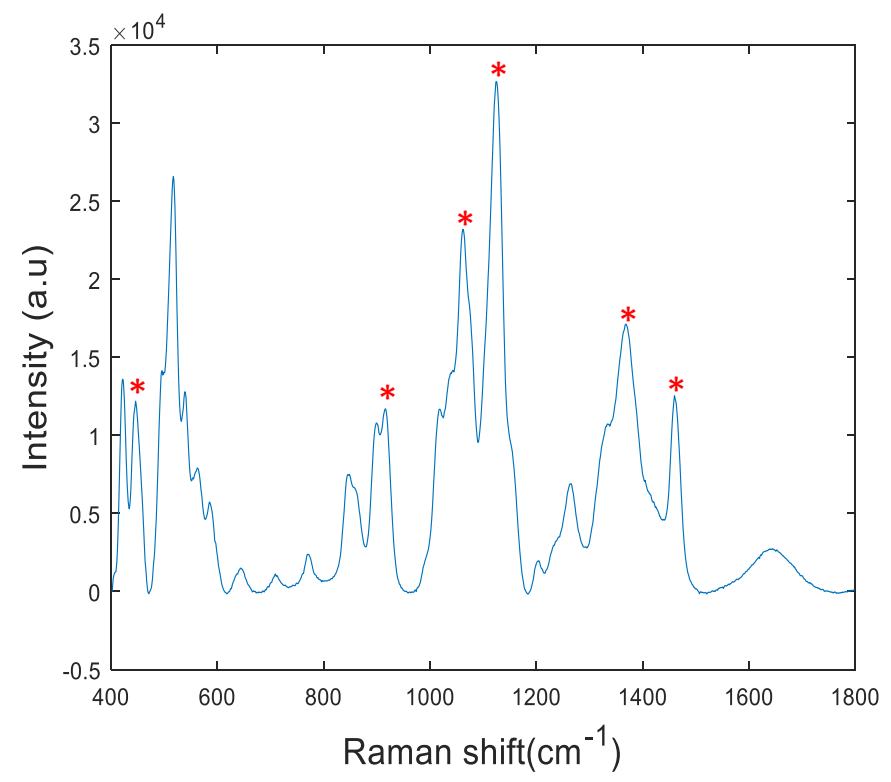

Fig.1. Raman spectrum of an aqueous glucose solution, concentration 450g/L. Example signature peaks at $450 \mathrm{~cm}^{-1}, 911 \mathrm{~cm}^{-1}, 1125 \mathrm{~cm}^{-1}, 1340 \mathrm{~cm}^{-1}$ and $1460 \mathrm{~cm}^{-1}$ are labelled in the figure.

\subsection{Monitoring the concentration dependence of glucose in distilled water}

In order to establish the analysis protocol for the patient samples, a PLSR prediction model was first built and applied to the set of varying concentrations of glucose in distilled water, as well as spiked into serum. The first step in this study was to optimise the measurement protocol and also to evaluate the efficacy of the centrifugal filtration technique in separating and concentrating glucose from the HMWF proteins. For this, different amounts of pure glucose were spiked into the distilled water. The normal glucose concentration and the concentration and hyper-glycaemia were deliberately included to simulate physiologically relevant concentrations. Figure 2 A displays examples of the EMSC (polynomial order 5) corrected low $(5 \times 100 \mathrm{mg} / \mathrm{dL})$, medium $(5 \times 450 \mathrm{mg} / \mathrm{dL})$ and high $(5 \times 1000 \mathrm{mg} / \mathrm{dL})$ datasets, showing glucose features (indicated by asterisks) of greater intensity at higher concentration, whereas the lower concentrations shows weaker features of glucose. The groups of spectra are off set for clarity. 
The concentration $1000 \mathrm{mg} / \mathrm{dL}$ was deliberately included in the dataset to evaluate the consistency of the glucose spectral features as the concentration increases from $100 \mathrm{mg} / \mathrm{dL}$ to $1000 \mathrm{mg} / \mathrm{dL}$. In order to analyse the spectral variations and the glucose concentrations, the PLSR algorithm was applied.
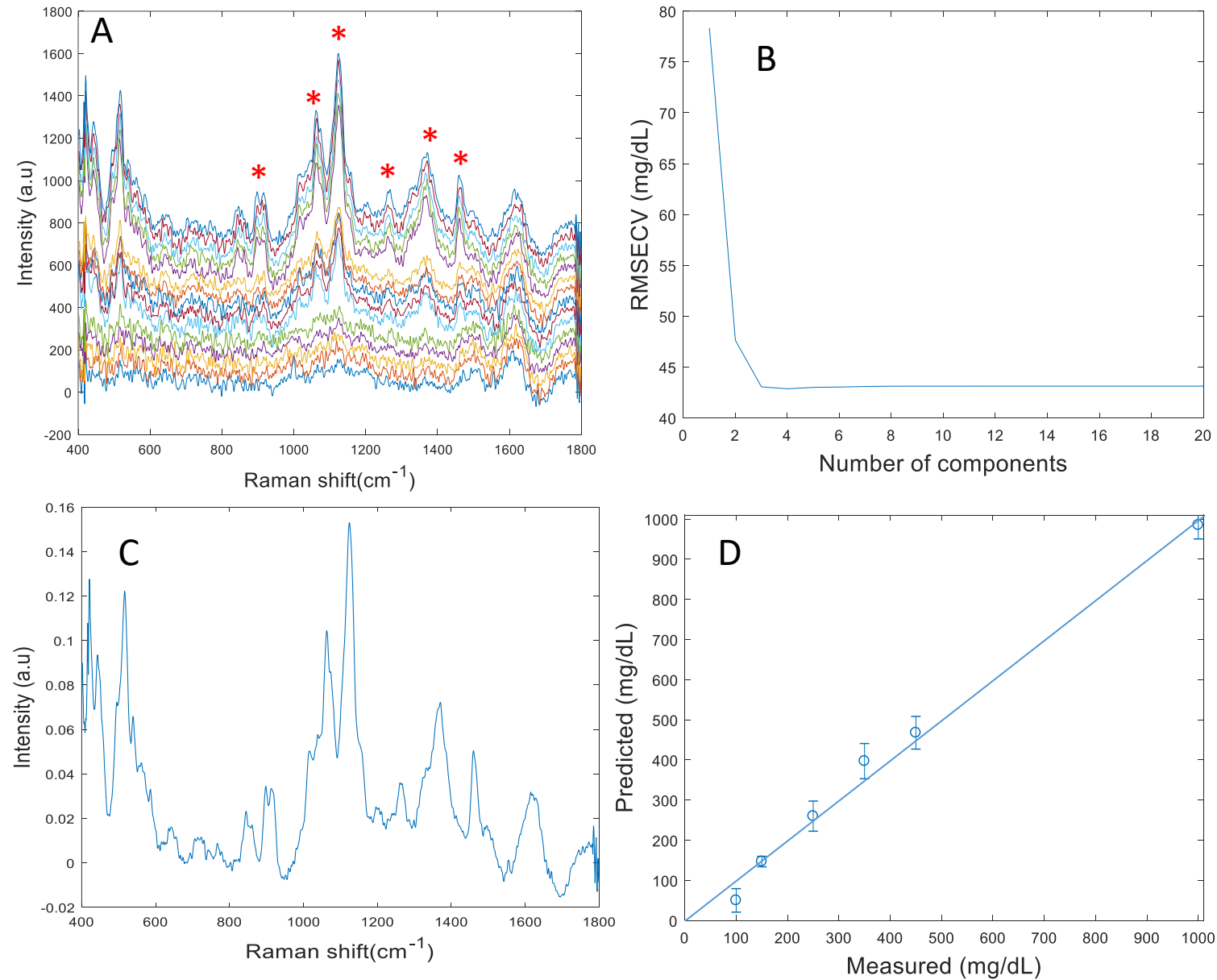

Fig. 2. (A): EMSC corrected Raman spectra of filtrate obtained after centrifugal filtration with $10 \mathrm{kDa}$ filters of varying concentrations of glucose $(5 \times 100 \mathrm{mg} / \mathrm{dL}, 5 \times 450 \mathrm{mg} / \mathrm{dL}$ and $5 \mathrm{x}$ $1000 \mathrm{mg} / \mathrm{dL}$, offset for clarity), in distilled water and signature peaks of glucose are highlighted with asterisks, (B): Evolution of the RMSECV on the validation model, (C): plot of PLSR coefficient with glucose features, (D): Predictive model built from the PLSR analysis. The value displayed in the PLSR model is an average of the concentration predicted with the corresponding standard deviation calculated from the 20 iterations of the cross validation. The RMSECV and $\mathrm{R}^{2}$ values were calculated as $10.93 \mathrm{mg} / \mathrm{dL}$ and 0.9705 respectively. 
Based on the percent variance explained by the latent variables and the minimum value of RMSECV (Figure 2B), the optimum number of latent variables to reach the best performance is determined to be 4 . The PLSR coefficient plot displayed in Figure $2 \mathrm{C}$ confirms the correlation of the data in Figure 2D is based on glucose features, such as the peaks at $\sim 1060 \mathrm{~cm}^{1}$, $\sim 1125 \mathrm{~cm}^{1}, 1450 \mathrm{~cm}^{-1}$ and $\sim 1340 \mathrm{~cm}^{-1}$. Finally, after selecting the optimum number of components for the data set analysed, a predictive model is built from the PLSR analysis (Figure 2D), to compare the observations to the known concentrations of glucose in the samples with the estimated concentrations from the spectral data sets. Figure 2D indicates that a satisfactory linear model could be obtained with the raw data set and that the concentration dependence of the sample set is conserved by centrifugal filtration. From the results shown in figure 2, it is evident that the predicted values are in good agreement with the reference concentrations and the corresponding correlation coefficient $\left(\mathrm{R}^{2}\right)$ is calculated as 0.9705 . Note, each concentration point has five independent measurements, and the mean standard deviation of each measurement is $4.8 \mathrm{mg} / \mathrm{dL}$. The RMSECV calculated from the 20 iteration of cross validation is $10.93 \mathrm{mg} / \mathrm{dL}$, thereby indicating that PLSR provides accurate predictions for the glucose Raman data over the entire concentration range of $100 \mathrm{mg} / \mathrm{dL}$ to $1000 \mathrm{mg} / \mathrm{dL}$.

\subsection{Monitoring the glucose concentration in spiked serum}

In an attempt to extend the optimised protocol to a more complex environment, PLSR analysis was performed on the EMSC corrected data set recorded from the filtrate of the serum samples with spiked glucose concentrations varying from $0 \mathrm{mg} / \mathrm{dL}$ to $220 \mathrm{mg} / \mathrm{dL}$. When the entire fingerprint region was selected for PLSR analysis, the resultant PLSR coefficient displayed a negative peak at $\sim 1000 \mathrm{~cm}^{-1}$ which could potentially derive from other LMWF species such as urea (63) (Figure S1C in Supplemental). Therefore, the spectral region from $1030 \mathrm{~cm}^{-1}$ to 
$1400 \mathrm{~cm}^{-1}$, which contains strong glucose features at $\sim 1050 \mathrm{~cm}^{-1}$ and at $1340 \mathrm{~cm}^{-1}$ but minimal interference from urea, has been chosen for PLSR analysis (62).
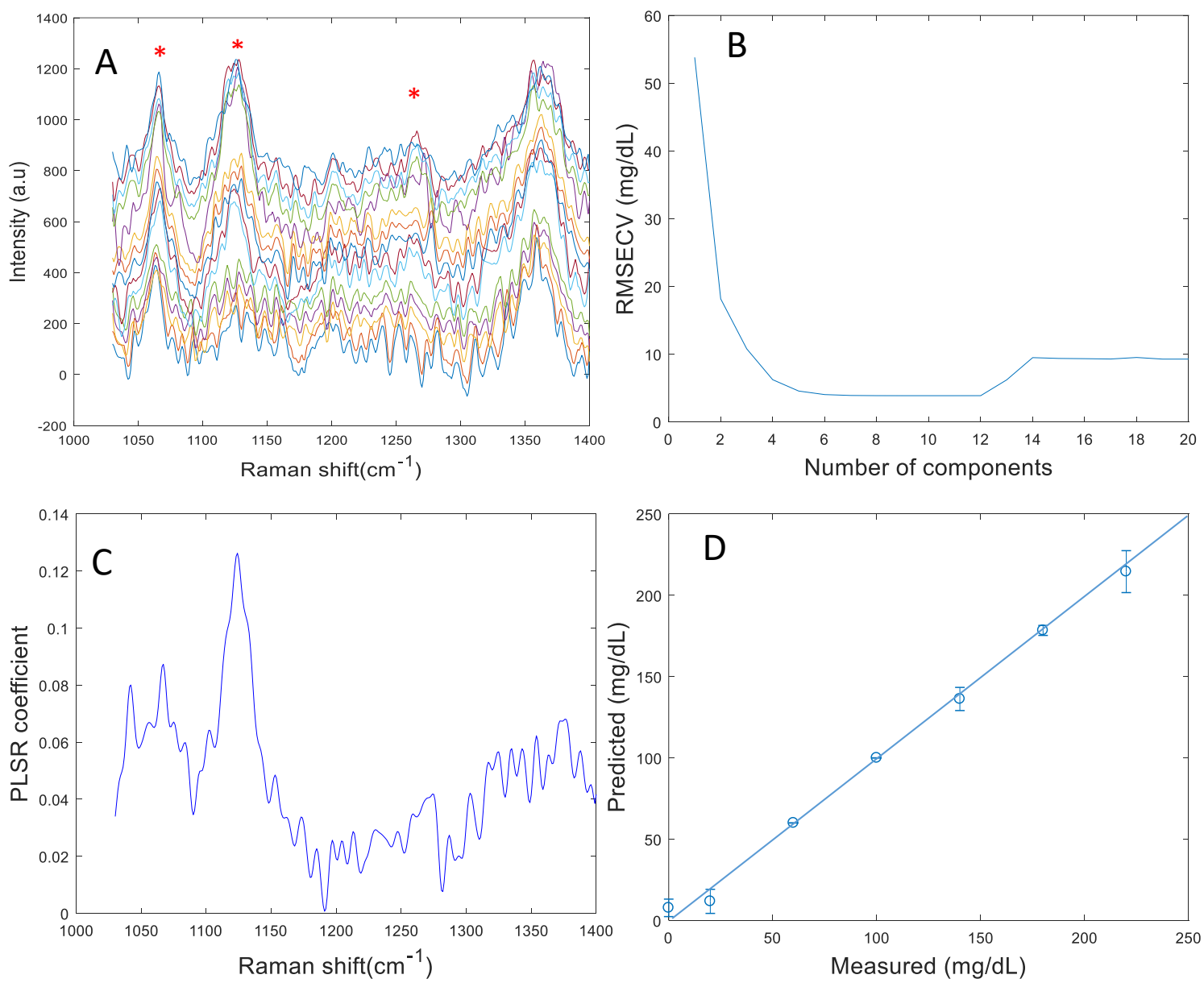

Fig. 3. (A): EMSC corrected Raman spectra of filtrate obtained after centrifugal filtration with $10 \mathrm{kDa}$ filters of glucose spiked in serum (spiked concentrations $5 \times 0 \mathrm{mg} / \mathrm{dL}, 5 \times 120 \mathrm{mg} / \mathrm{dL}$ and $5 \times 220 \mathrm{mg} / \mathrm{dL}$, offset for clarity) and the signature peaks of glucose are highlighted by asterisks, (B): Evolution of RMSECV of the data set (C): plot of PLSR coefficient with glucose features, (D): Predictive model built from the PLSR analysis. The value displayed in the PLSR model is an average of the concentration predicted with the corresponding standard deviation calculated from the 20 iterations of the cross validation. The RMSECV and $\mathrm{R}^{2}$ values were calculated as $1.66 \mathrm{mg} / \mathrm{dL}$ and 0.9914 
Figure 3A shows the glucose data set after background correction using the EMSC algorithm (offset for clarity by 5.0 units). The optimum number of latent variables were chosen by calculating the lowest value of RMSECV (Figure 3B). Six latent variables were chosen for this model and the resultant PLSR coefficient exhibits strong glucose features, as shown in Figure 3C. A linear predictive model can be defined from the EMSC corrected data set of varying concentration of glucose in serum Figure 3D. The RMSECV was found to be $1.66 \mathrm{mg} / \mathrm{dL}$ and $\mathrm{R}^{2}$ value was calculated as 0.9914 . The mean standard deviation of each measurement from 20 iterations of cross validation was calculated to be $3.2 \mathrm{mg} / \mathrm{dL}$. The results suggest that this optimised protocol can be applied to the patient samples to build a quantitative model.

Bonnier et al. have previously illustrated the strategy of centrifugal filtration to fractionate the samples to eliminate the influence of HMW in order to screen potential LMWF biomarkers using glucose as a model analyte, measured by ATR-FTIR [33]. The results of the analysis of similarly centrifugally filtered, glucose spiked samples of commercial serum are reproduced in Table 2, and directly compared to the results of the current study using Raman spectroscopic analysis in the native liquid state. Although each method yields similar $\mathrm{R}^{2}$ values, the RMSECV of Raman spectroscopic analysis is significantly lower, which indicates an increased accuracy of the measurement protocol in the liquid state.

Table 2. Comparison of the results of ATR-FTIR and Raman spectroscopic analysis of human serum spiked with varying concentrations of glucose.

\begin{tabular}{|c|c|c|c|c|}
\hline $\begin{array}{c}\text { Measurement } \\
\text { type }\end{array}$ & $\begin{array}{c}\text { Concentration } \\
\text { range (mg/dL) }\end{array}$ & RMSECV(mg/dL) & $\begin{array}{c}\text { Standard } \\
\text { deviation }\end{array}$ & $\mathrm{R}^{2}$ \\
\hline $\begin{array}{c}\text { FTIR (Min- } \\
\text { Max } \\
\text { normalised) }\end{array}$ & $0-220$ & 2.199 & 0.250 & 0.995 \\
\hline $\begin{array}{c}\text { Raman } \\
\text { spectroscopy }\end{array}$ & $0-220$ & 1.665 & 3.2 & 0.991 \\
\hline
\end{tabular}




\subsection{Monitoring the glucose concentration in patient samples}

Figure 4A displays the Raman spectra from patient samples after performing background correction using the EMSC algorithm, in the reduced spectral range of $1030-1400 \mathrm{~cm}^{-1}$. The spectra are offset for clarity by 5.0 units. The glucose bands at $1340 \mathrm{~cm}^{-1}$ and $1460 \mathrm{~cm}^{-1}$, related to the $\delta(\mathrm{C}-\mathrm{C}-\mathrm{H})$ vibration and pure $\mathrm{CH}_{2}$ group vibration, and the peak at $1060 \mathrm{~cm}^{-1}$ due to $v(\mathrm{C} 1-$ $\mathrm{OH})$ stretching can be clearly seen. Based on the minimum RMSECV value (Figure 4B), 12 latent variables were found to be optimal for constructing a PLSR based model. The PLSR coefficient clearly shows glucose features (Figure $4 \mathrm{C}$ ), indicating that the prediction is based on the variation in the glucose peak intensities. In the prediction model of Figure 4D, the minimum value of RMSECV is $1.84 \mathrm{mg} / \mathrm{dL}$ and the $\mathrm{R}^{2}$ value is calculated as 0.82 , indicating a high prediction capacity. The mean standard deviation was determined to be $3.48 \mathrm{mg} / \mathrm{dL}$, indicating acceptable repeatability between the cross validation iterations. 

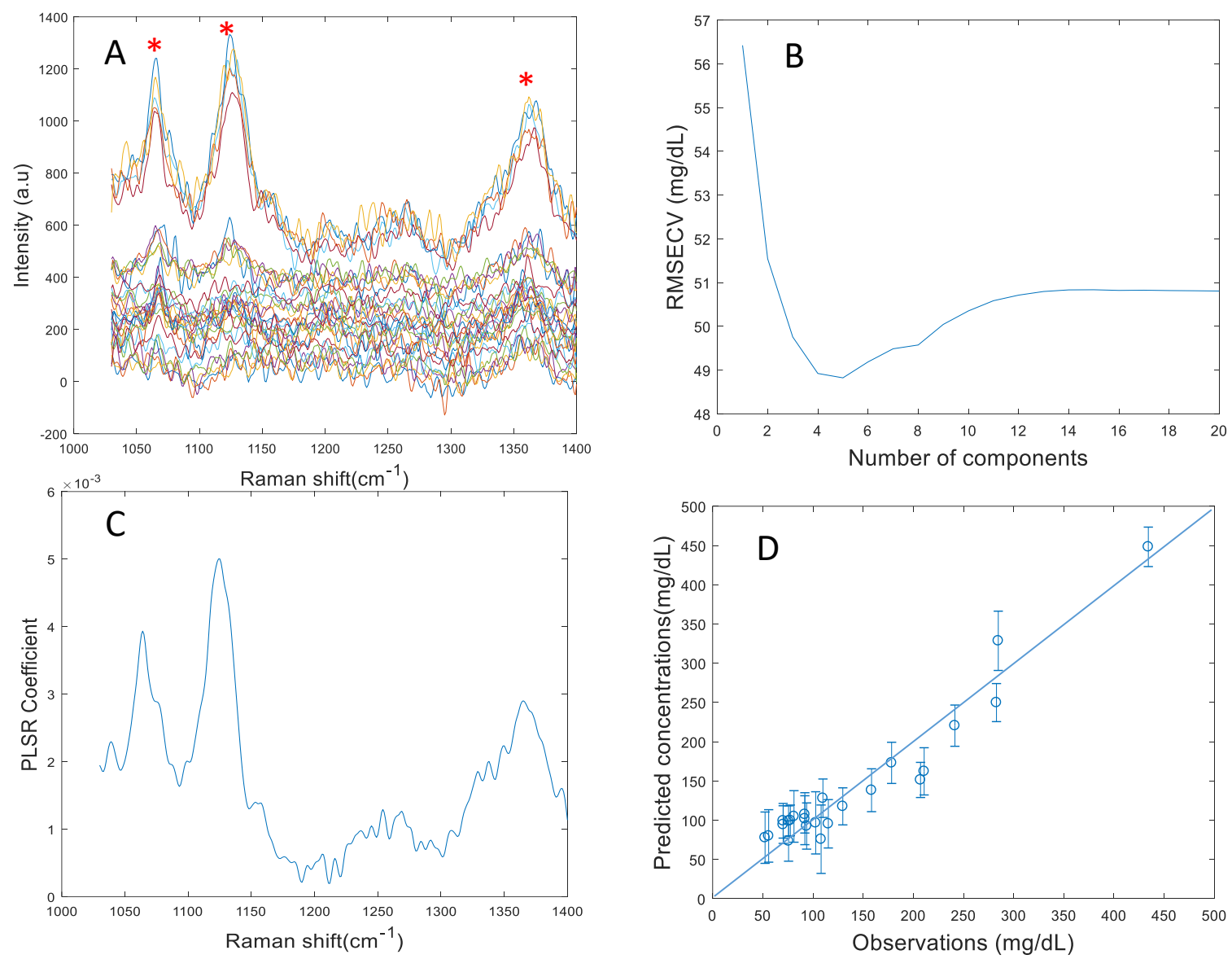

Fig. 4. (A): EMSC corrected Raman spectra of filtrate obtained after centrifugal filtration with $10 \mathrm{kDa}$ filters of patient samples $(5 \times 52.25 \mathrm{mg} / \mathrm{dL}, 5 \times 75.67 \mathrm{mg} / \mathrm{dL}, 5 \times 93.69 \mathrm{mg} / \mathrm{dL}, 5 \times$ $210.81 \mathrm{mg} / \mathrm{dL}$ and $5 \times 434.35 \mathrm{mg}$, offset for clarity) and the signature peaks are marked by asterisks, (B): Evolution of RMSECV of the data set, (C): plot of PLSR coefficient with glucose features, (D): Predictive model built from the PLSR analysis. The value displayed in the PLSR model is an average of the concentration predicted with the corresponding standard deviation calculated from the 20 iterations of the cross validation The RMSECV and $\mathrm{R}^{2}$ values were calculated as $1.84 \mathrm{mg} / \mathrm{dL}$ and 0.84 respectively.

Table 3. Comparison of the results of ATR-FTIR (29) and Raman spectroscopic analysis of patient sample set for monitoring the glucose levels. FTIR results are normalised.

\begin{tabular}{|l|l|l|l|l|}
\hline $\begin{array}{l}\text { Measurement } \\
\text { type }\end{array}$ & $\begin{array}{l}\text { Concentration } \\
\text { range }(\mathrm{mg} / \mathrm{dL})\end{array}$ & RMSECV(mg/dL) & $\begin{array}{l}\text { Standard } \\
\text { deviation(mg/dL) }\end{array}$ & $\mathrm{R}^{2}$ \\
\hline $\begin{array}{l}\text { ATR-FTIR } \\
\text { (Min-Max } \\
\text { normalised) }\end{array}$ & $61.25-210$ & 3.1 & 1.90 & 0.9957 \\
\hline
\end{tabular}




\begin{tabular}{|l|l|l|l|l|}
\hline $\begin{array}{l}\text { Raman } \\
\text { Spectroscopy }\end{array}$ & $52.25-210$ & 1.6 & 2.31 & 0.91 \\
& & & & \\
\cline { 2 - 5 } & $52.25-440$ & 1.84 & 3.48 & 0.84 \\
\hline
\end{tabular}

Table 3 directly compares the results of analysis of a similar patient sample-set, with glucose levels which varied over the range $0-210 \mathrm{mg} / \mathrm{dL}$, similarly centrifugally filtered and analysed using ATR-FTIR (29) and the results of the current study of patient samples over the same (patients 1-21, Table 1) and extended (patients 1-25, Table 1) range using Raman spectroscopic analysis in the native liquid state. It is noteworthy that Raman spectroscopy yields significantly lower values of RMSECV for all the Raman prediction models, suggesting higher sensitivity and accuracy. However, the standard deviation was found to be higher and the $\mathrm{R}^{2}$ value was found to be considerably lower for the shorter as well as larger concentration range. The reduced $R^{2}$ value and higher standard of deviation could be attributed to the variability in the spectral response of the patient samples. Nevertheless, the precision of the model expressed by the RMSECV values indicates the suitability of this technique to discriminate patients with very close concentrations of blood glucose. The results demonstrate that Raman spectroscopy is able to detect subtle variations in the glucose concentrations with similar accuracy in the native liquid state, to the ATR-FTIR method in dried samples.

In order for a glucose detection method to be viable, it should be able to detect glucose in the clinically relevant range $(10-450 \mathrm{mg} / \mathrm{dL})$. Post PLSR analysis, the dataset is presented in the Clarke's error grid (Figure 5), the most common standard for evaluating the performance of a glucose detection method used since 1987 (64). Data points that fall in zone A and B are acceptable values. Values that fall outside A and B result in erroneous diagnosis. On the Clarke's error grid of patient samples (Figure 5), 98\% of the PLSR validation dataset falls 
within zone $\mathrm{A}$ and $\mathrm{B}$, which is the zone of clinical accurate measurement with no effect on clinical actions. Error can be attributed to the intrinsic variability of patient samples which reflects their physiological state on the day. However, the results from this study are promising, indicating that Raman spectroscopy coupled with multivariate analysis and centrifugal filtration techniques can be used as a biochemical tool for detecting potential small biomarkers from human serum/plasma.

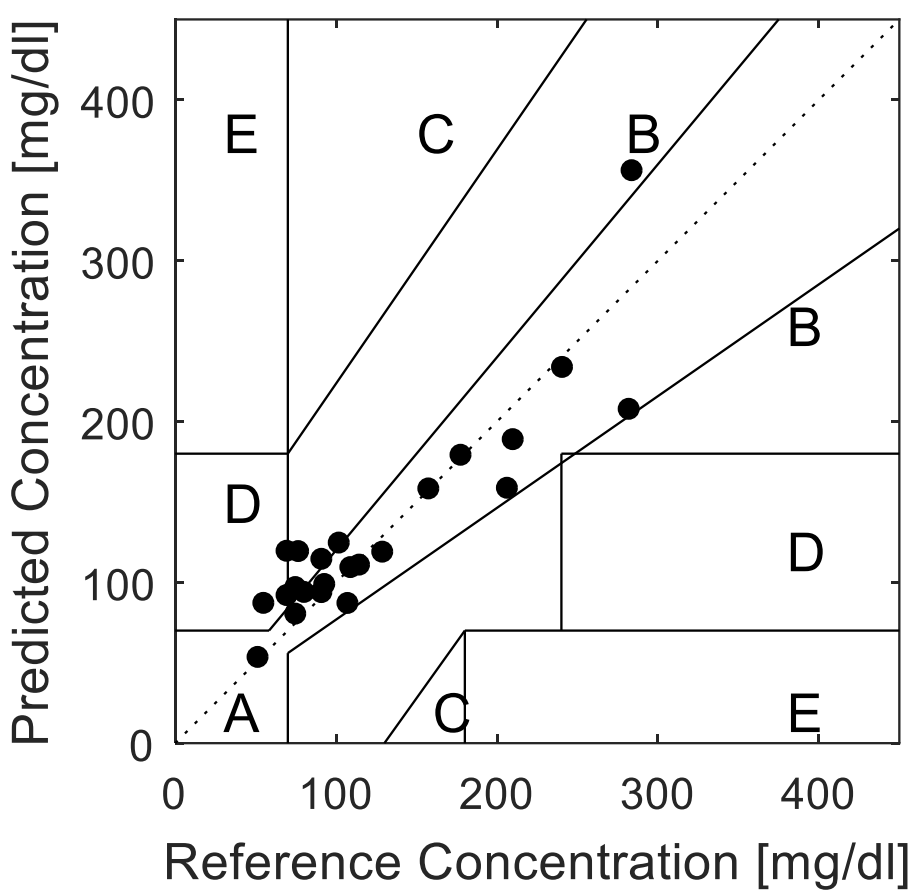

Fig.5. PLSR validation of patient samples on Clarke's error grid. The RMSECV was found to be $1.84 \mathrm{mg} / \mathrm{dL}$ and $\mathrm{R}^{2}$ value was calculated as 0.84

\section{Discussion}

The potential advantages of using vibrational spectroscopy for biomarker assessment in bodily fluids have been extensively explored in the last two decades. However, little consideration has been given so far to protocols involving Raman analysis in the native liquid state of proteins. Liakat et al. reported the in vitro prediction of physiologically relevant concentration of glucose using mid-IR transmission light with respect to a Clarke error grid (52). Near IR and Raman 
spectroscopy was also used for measurement of glucose from artificial plasma with high precision and accuracy (65). Although these are initial steps towards developing Raman spectroscopy into a biochemical tool for serum/plasma analysis, measurements should be performed on patient samples in order to ensure the relevancy of these methods. Using Raman spectroscopy as a biochemical tool, it has been possible to detect differences in peak intensities of altered serum compared to normal ones for glucose and lipid compounds (66), multicomponent blood analysis (67) and also to determine blood glucose concentration of blood samples with above-physiological levels of glucose within 5 min (68).

Using glucose as a model analyte, this study successfully demonstrated the feasibility of employing Raman spectroscopy for detecting small biomarkers in serum after depletion of HMWF proteins. It has been shown that optimal experimental set up for Raman analysis for this experiment is Lab-Tek plates as substrate and measurement in the inverted geometry using water immersion objective and the sample volume can be as small as $1 \mu \mathrm{L}$. This experimental set up is advantageous for clinical purposes where the volumes of patient samples are minimal. After the depletion of the abundant proteins, the dominant water peak from the filtrate collected after centrifugal filtration using $10 \mathrm{kDa}$ can be removed by using the EMSC algorithm, and PLSR analysis applied to obtain a prediction model relating the glucose concentrations and the intensity of glucose features. Even though the EMSC algorithm removed the underlying water spectra effectively, there could be interference from other LMWF analytes, namely, urea (7$20 \mathrm{mg} / \mathrm{dL})$. Thus, as presented in the present study, the spectral range from $1030 \mathrm{~cm}^{-1}$ to $1400 \mathrm{~cm}^{-}$ ${ }^{1}$ was chosen for data analysis, as this region does not contain signature peaks of urea.

The depletion of HMWF proteins using centrifugal filtration to detect glucose in serum using ATR-FTIR was previously reported by Bonnier et al. (29). While the work carried out by Bonnier et al. showed excellent results, the requirement of a drying step is potentially a major drawback. Indeed, the drying process required for ATR-FTIR has been identified by Cameron 
et al. as a potentially significant impediment to translation of the technique to clinical applications (41). Since Raman is compatible with aqueous samples, sample drying can be avoided and data can be recorded from the native environment, which makes the proposed method an ideal alternative to IR. The results summarised in Table 2 and Figure 3 suggest that Raman spectroscopy maintained high level of accuracy and predictive power and the relationship between spectral variation and protein concentrations is linear, with minimal standard deviation. The PLSR model built on varying concentration of glucose spiked in serum provided an accurate prediction model $\left(\mathrm{R}^{2}=0.9914, \mathrm{RMSECV}=1.66 \mathrm{mg} / \mathrm{dL}\right)$ after applying preprocessing steps using the EMSC based algorithm. Having established the optimal sample preparation and analysis protocol using the spiked serum model, the same protocol was applied to patient samples. In the case of patient samples, RMSECV and $\mathrm{R}^{2}$ values were calculated to be $1.84 \mathrm{mg} / \mathrm{dL}$, and 0.84 respectively. Although ATR-FTIR provides better standard deviation and higher $\mathrm{R}^{2}$ value, the Raman prediction model gives lower a RMSECV value, indicating higher accuracy. The PLSR coefficient plot shows clear features of glucose, indicating the prediction model is built on variations in glucose concentrations. The added advantage of Raman spectroscopy is that the analysis can be performed on liquid samples and no additional time delays associated with sample drying are introduced. Although the quantitative capability of Raman can be easily demonstrated using glucose spiked with a pooled serum model, the analysis of patient samples can be more complicated, the reason being the intrinsic variability of individual samples depending upon the physiological state of the individual on that day.

For the purposes of a direct comparison of the two techniques, glucose concentration in human serum was chosen, and the results detailed in Table 3 indicate that Raman in the liquid state provides higher accuracy than FTIR-ATR. Although vibrational spectroscopic techniques are unlikely to replace current techniques for routine glucose monitoring, in a more general sense, an argument for serological applications of vibrational spectroscopic techniques has been made 
$(28,37)$. The advantages of using Raman spectroscopy over common biochemical assays to quantify urea and creatinine has previously been reported(63). Moreover, Raman spectroscopy as a biochemical tool for serum analysis is cost effective, rapid and a non-destructive method as compared to currently employed gold standard clinical methods such as spectrophotometric analysis ( e.g. COBAS analyser) (69). The COBAS analyser has a standard deviation of 0.04 mmol. $\mathrm{L}^{-1}$ (which is equivalent to $0.721 \mathrm{mg} \cdot \mathrm{dL}^{-1}$ ), as mentioned in the Material and Methods section, and the lower detection limit is $2 \mathrm{mg} / \mathrm{dL}$ (70) with a correlation coefficient of 0.975 reported for immunoassays (71) and $\mathrm{R}^{2}$ of 0.990 for urea, creatinine, sugar, total protein and calcium (72). Similar $\mathrm{R}^{2}$ values were calculated for pure glucose solutions and glucose spiked serum solutions using Raman spectroscopy, suggesting that Raman spectroscopy is well-suited for routine use as a biochemical tool for glucose analysis. However, the analysis using COBAS analyser is complex and uses various enzymatic reagents, increasing the cost and chance of inaccuracy in the results obtained. Employing well trained personnel to operate the equipment enhances the reliability but increases the cost. Hence, Raman spectroscopy offers several advantages as it is a onetime investment, easy to operate and provides rapid results with wider information without destroying the medium. This could be translated as an alternative method for glucose monitoring, especially in the case of hyperglycaemia. Further studies need to be conducted to investigate other LMWF metabolites from human serum using Raman spectroscopy. However, to further ensure relevancy of the results, the study should ultimately be conducted on large number of patient samples.

\section{Conclusion:}

Summarising, the work presented showcases the development of an optimal methodology for the detection of LMWF analytes from human serum using Raman spectroscopy with minimal sample preparation steps and without the use of any extrinsic labels. The proposed approach can be expeditiously employed for early detection of pathological disorders associated with 
high or low serum/plasma proteins/biomarkers. Disease diagnosis from bodily fluids can be developed into a dynamic diagnostic environment that will enable early disease diagnosis even before the disease becomes symptomatic. Thus, analysis of bodily fluids has emerged as one of the promising approaches to deliver crucial information about patient health and monitor disease progression and/or therapy. Given the remarkable advances in the field over the last two decades, including sample preparation, protein fractionation, quantitation and chemometrics, it is conceivable that vibrational spectroscopic techniques can be developed as a point-of-care disease monitoring system. Ultimately, the proof of concept presented in this study can be easily transferable to any other low molecular weight biomarkers or therapeutic drugs.

\section{Conflicts of Interest}

There are no conflicts to declare.

\section{Acknowledgements}

Drishya Rajan Parachalil was funded by DIT Fiosraigh scholarship. J. McIntyre was funded by Science Foundation Ireland, PI/11/08. The Irish-French PHC Ulysses 2018 Collaborative project is jointly funded by the Irish Research Council and Campus France with participation of the French embassy in Ireland.

\section{References}

1. Veenstra TD, Conrads TP, Hood BL, Avellino AM, Ellenbogen RG, Morrison RS. Biomarkers: Mining the Biofluid Proteome. Mol Cell Proteomics. 2005;4(4):409-18.

2. Kong K, Kendall C, Stone N, Notingher I. Raman spectroscopy for medical diagnostics - From in-vitro biofluid assays to in-vivo cancer detection. Adv Drug Deliv Rev. 2015;89:121-34.

3. Su S-B, Chuen T, Poon W, Thongboonkerd V. Human Body Fluid. Biomed Res Int. 2013;2013:2-4.

4. Busher JT. Serum Albumin and Globulin. Clin Methods Hist Phys Lab Exam. 
1990;497-9.

5. Greening DW, Simpson RJ. Low-Molecular Weight Plasma Proteome Analysis Using Centrifugal Ultrafiltration. Methods Mol Biol. 2011;728:109-24.

6. Parker CE, Borchers $\mathrm{CH}$. Mass spectrometry based biomarker discovery, verification, and validation--Quality assurance and control of protein biomarker assays. Mol Oncol. 2014;8(4):840-58.

7. Baker MJ, Hughes CS, Hollywood KA. Biophotonics: Vibrational Spectroscopic Diagnostics. Morgan \& Claypool Publishers; 2016.

8. Li J, Zhang Z, Rosenzweig J, Wang YY, Chan DW. Proteomics and Bioinformatics Approaches for Identification of Serum Biomarkers to Detect Breast Cancer. Clin Chem. 2002 Aug;48(8):1296-304

9. Bast RC, Ravdin P, Hayes DF, Bates S, Fritsche H, Jessup JM, et al. 2000 Update of Recommendations for the Use of Tumor Markers in Breast and Colorectal Cancer: Clinical Practice Guidelines of the American Society of Clinical Oncology. J Clin Oncol. 2001 Mar 15;19(6):1865-78.

10. Hormigo A, Gu B, Karimi S, Riedel E, Panageas KS, Edgar MA, et al. YKL-40 and matrix metalloproteinase-9 as potential serum biomarkers for patients with high-grade gliomas. Clin Cancer Res. 2006 Oct;12(19):5698-704.

11. Labrie F, Dupont A, Suburu R, Cusan L, Tremblay M, Gomez JL, et al. Serum prostate specific antigen as pre-screening test for prostate cancer. J Urol. $1992 \mathrm{Mar}$;147(3 Pt 2):842-6.

12. Catalona WJ, Smith DS, Ratliff TL, Dodds KM, Coplen DE, Yuan JJ, et al. Measurement of prostate-specific antigen in serum as a screening test for prostate cancer. N Engl J Med. 1991 Apr;324(17):1156-61.

13. Locker GY, Hamilton S, Harris J, Jessup JM, Kemeny N, Macdonald JS, et al. ASCO 2006 update of recommendations for the use of tumor markers in gastrointestinal cancer. J Clin Oncol. 2006 Nov;24(33):5313-27.

14. Liesenfeld DB, Habermann N, Owen RW, Scalbert A, Ulrich CM. Review of mass spectrometry-based metabolomics in cancer research. Cancer Epidemiol Biomarkers Prev. 2013;22(12):2182-201.

15. Kimhofer T, Fye H, Taylor-Robinson S, Thursz M, Holmes E. Proteomic and metabonomic biomarkers for hepatocellular carcinoma: A comprehensive review. $\mathrm{Br} \mathbf{J}$ Cancer. 2015;112(7):1141-56.

16. Nowak M, Janas Ł, Stachowiak G, Stetkiewicz T, Wilczyński JR. Current clinical application of serum biomarkers to detect ovarian cancer. Prz Menopauzalny. 2015;14(4):254-9.

17. Luque-Garcia JL, Neubert TA. Sample preparation for serum/plasma profiling and biomarker identification by mass spectrometry. J Chromatogr A. 2007;1153(1-2):25976.

18. Fernandez-Olavarria A, Mosquera-Perez R, Diaz-Sanchez R, Serrera-Figallo M, Gutierrez-Perez J, Torres-Lagares D. The role of serum biomarkers in the diagnosis and prognosis of oral cancer: A systematic review. J Clin Exp Dent. 2016;8(2):184- 
193.

19. Sahab ZJ, Semaan SM, Sang Q-XA. Methodology and applications of disease biomarker identification in human serum. Biomark Insights. 2007;2:21-43.

20. Adkins JN, Varnum SM, Auberry KJ, Moore RJ, Angell NH, Smith RD, et al. Toward a Human Blood Serum Proteome. Mol Cell Proteomics. 2002;1(12):947-55.

21. Lundblad RL. Considerations for the use of blood plasma and serum for proteomic analysis. Internet J Genomics Proteomics. 2005;1(2):1-8.

22. Pieper R, Gatlin CL, Makusky AJ, Russo PS, Schatz CR, Miller SS, et al. The human serum proteome: Display of nearly 3700 chromatographically separated protein spots on two-dimensional electrophoresis gels and identification of 325 distinct proteins. Proteomics. 2003;3(7):1345-64.

23. Lacombe C, Untereiner V, Gobinet C, Zater M, Sockalingum GD, Garnotel R. Rapid screening of classic galactosemia patients: A proof-of-concept study using highthroughput FTIR analysis of plasma. Analyst. 2015;140(7):2280-6.

24. Di Girolamo F, Alessandroni J, Somma P, Guadagni F. Pre-analytical operating procedures for serum Low Molecular Weight protein profiling. J Proteomics. 2010;73(3):667-77.

25. Patel KN, Patel JK, Patel MP, Rajput GC, Patel HA. Introduction to hyphenated techniques and their applications in pharmacy. Pharm Methods. India: Medknow Publications \& Media Pvt Ltd; 2010;1(1):2-13.

26. Kuligowski J, Cascant M, Garrigues S, De La Guardia M. An infrared spectroscopic tool for process monitoring: Sugar contents during the production of a depilatory formulation. Talanta. 2012;99:660-7.

27. Edelmann A, Diewok J, Baena JR, Lendl B. High-performance liquid chromatography with diamond ATR-FTIR detection for the determination of carbohydrates, alcohols and organic acids in red wine. Anal Bioanal Chem. 2003;376(1):92-7.

28. Ioannou A. Real Time Monitoring the Maillard Reaction Intermediates by HPLCFTIR. J Phys Chem Biophys. 2016;6(2):6-10.

29. Bonnier F, Blasco H, Wasselet C, Brachet G, Respaud R, Carvalho LFCS, et al. Ultrafiltration of human serum for improved quantitative analysis of low molecular weight biomarkers using ATR-IR spectroscopy. Analyst. 2017;142(8):1285-98.

30. Byrne H, Sockalingum G, Stone N. Raman Microscopy: Complement or Competitor. Biomed Appl Synchrotron Infrared Microspectrosc. 2011;(11):105-42.

31. Bonnier F, Petitjean F, Baker MJ, Byrne HJ. Improved protocols for vibrational spectroscopic analysis of body fluids. J Biophotonics. 2014;7(3-4):167-79.

32. Bonnier F, Baker MJ, Byrne HJ. Vibrational spectroscopic analysis of body fluids: avoiding molecular contamination using centrifugal filtration. Anal Methods. 2014;6(14):5155.

33. Bunaciu AA, Fleschin Ş, Hoang VD, Aboul-Enein HY. Vibrational Spectroscopy in Body Fluids Analysis. Crit Rev Anal Chem. 2017;47(1):67-75.

34. Mitchell AL, Gajjar KB, Theophilou G, Martin FL, Martin-Hirsch PL. Vibrational 
spectroscopy of biofluids for disease screening or diagnosis: Translation from the laboratory to a clinical setting. J Biophotonics. 2014;7(3-4):153-65.

35. Nyuwi KT, Gyan Singh CH, Khumukcham S, Rangaswamy R, Ezung YS, Chittvolu $\mathrm{SR}$, et al. The role of serum fibrinogen level in the diagnosis of acute appendicitis. $\mathrm{J}$ Clin Diagnostic Res. 2017;11(1):PC13-PC15.

36. Tekin IO, Pocan B, Borazan A, Ucar E, Kuvandik G, Ilikhan S, et al. Positive correlation of CRP and fibrinogen levels as cardiovascular risk factors in early stage of continuous ambulatory peritoneal dialysis patients. Ren Fail. 2008;30(2):219-25.

37. Stec JJ, Silbershatz H, Tofler GH, Matheney TH, Sutherland P, Lipinska I, et al. Association of fibrinogen with cardiovascular risk factors and cardiovascular disease in the Framingham Offspring Population. Circulation. 2000;102(14):1634-8.

38. Ariëns RAS. Elevated fibrinogen causes thrombosis. Blood. 2011;117(18): 4687-8.

39. Hong LF, Li XL, Luo SH, Guo YL, Zhu CG, Qing P, et al. Association of fibrinogen with severity of stable coronary artery disease in patients with type 2 diabetic mellitus. Dis Markers. 2014;2014:485687.

40. Paraskevaidi M, Morais CLM, Lima KMG, Snowden JS, Saxon JA. Differential diagnosis of Alzheimer' $s$ disease using spectrochemical analysis of blood. PNAS. 2017; 114 (38):929-38

41. Cameron JM, Butler HJ, Palmer DS, Baker MJ. Biofluid spectroscopic disease diagnostics: A review on the processes and spectral impact of drying. J Biophotonics. 2018;11(4):1-12.

42. Shaw RA, Kotowich S, Leroux M, Mantsch HH. Multianalyte Serum Analysis Using Mid-Infrared Spectroscopy. Ann Clin Biochem. 1998 Sep 1;35(5):624-32.

43. Gajjar K, Trevisan J, Owens G, Keating PJ, Wood NJ, Stringfellow HF, et al. Fouriertransform infrared spectroscopy coupled with a classification machine for the analysis of blood plasma or serum: a novel diagnostic approach for ovarian cancer. Analyst. 2013;138(14):3917-26.

44. Shaw RA, Low-Ying S, Man A, Liu K-Z, Mansfield C, Rileg CB, et al. Infrared Spectroscopy of Biofluids in Clinical Chemistry and Medical Diagnostics. In: Biomedical Vibrational Spectroscopy. John Wiley \& Sons, Inc.; 2007. p. 79-103.

45. Bonnier F, Brachet G, Duong R, Sojinrin T, Respaud R, Aubrey N, et al. Screening the low molecular weight fraction of human serum using ATR-IR spectroscopy. J Biophotonics. 2016;9(10):1085-97.

46. Spalding K, Bonnier F, Bruno C, Blasco H, Board R, Benz-de Bretagne I, et al. Enabling quantification of protein concentration in human serum biopsies using attenuated total reflectance - Fourier transform infrared (ATR-FTIR) spectroscopy. Vib Spectrosc. 2018;99:50-8.

47. Nabers A, Perna L, Lange J, Mons U, Schartner J, Güldenhaupt J, et al. Amyloid blood biomarker detects Alzheimer's disease. EMBO Mol Med. 2018;(May):1-11.

48. Roy S, Perez-Guaita D, Andrew DW, Richards JS, McNaughton D, Heraud P, et al. Simultaneous ATR-FTIR Based Determination of Malaria Parasitemia, Glucose and Urea in Whole Blood Dried onto a Glass Slide. Anal Chem. 2017 May 
16;89(10):5238-45.

49. Hands JR, Abel P, Ashton K, Dawson T, Davis C, Lea RW, et al. Investigating the rapid diagnosis of gliomas from serum samples using infrared spectroscopy and cytokine and angiogenesis factors. Anal Bioanal Chem. 2013;405(23):7347-55.

50. Parachalil DR, Brankin B, McIntyre J, Byrne HJ. Raman spectroscopic analysis of high molecular weight proteins in solution - considerations for sample analysis and data pre-processing. Analyst . 2018;143(24):5987-98.

51. Daines TL, Morse KW. Determination of Glucose in Blood Serum. J Chem Educ. 1976;53(2):126-7.

52. Liakat S, Bors KA, Huang T, Michel APM, Zanghi E, Gmachl CF, et al. In vitro measurements of physiological glucose concentrations in biological fluids using midinfrared light. Biomed Opt Express. 2013;4(7):233-9.

53. Illingworth J. Methods of enzymatic analysis: Third edition. Biochemical Education.1985;13(1):38

54. Bell C. Clinical Guide to Laboratory Tests. 3rd edition. Norbert W. Tietz, ed. Transfusion. Wiley/Blackwell; 2018 Sep 17;35(11):972.

55. Kerr LT, Hennelly BM. A multivariate statistical investigation of background subtraction algorithms for Raman spectra of cytology samples recorded on glass slides. Chemom Intell Lab Syst. 2016;158:61-8.

56. Kohler A, Kirschner C, Oust A, Martens H. Extended multiplicative signal correction as a tool for separation and characterization of physical and chemical information in Fourier transform infrared microscopy images of cryo-sections of beef loin. Appl Spectrosc. 2005 Jun;59(6):707-16.

57. Liland KH, Kohler A, Afseth NK. Model-based pre-processing in Raman spectroscopy of biological samples. J Raman Spectrosc. 2016;47(6):643-50.

58. Joss L, Müller EA. Machine Learning for Fluid Property Correlations: Classroom Examples with MATLAB. J Chem Educ. 2019; 0(0).

59. Hen XIS, Iang LXU, Hubin SYE, Ong RHU, In LINGJ, Anyang HXU, et al. Automatic baseline correction method for the open-path Fourier transform infrared spectra by using simple iterative averaging. Opt Expres. 2018;26(10):609-14.

60. Wold S, Sjöström M, Eriksson L. PLS-regression: A basic tool of chemometrics. Chemom Intell Lab Syst. 2001;58(2):109-30.

61. Russell S, Norvig P. Artificial Intelligence: A Modern Approach. 3rd ed. Upper Saddle River, NJ, USA: Prentice Hall Press; 2009.

62. Söderholm S, Roos YH, Meinander N, Hotokka M. Raman spectra of fructose and glucose in the amorphous and crystalline states. J Raman Spectrosc.

1999;30(11):1009-18.

63. Saatkamp CJ, de Almeida ML, Bispo JAM, Pinheiro ALB, Fernandes AB, Silveira L. Quantifying creatinine and urea in human urine through Raman spectroscopy aiming at diagnosis of kidney disease. J Biomed Opt. 2016;21(3):037001.

64. Clarke WL, Cox D, Gonder-Frederick LA, Carter W, Pohl SL. Evaluating Clinical 
Accuracy of Systems for Self-Monitoring of Blood Glucose. Diabetes Care. 1987 Sep 1;10(5):622 LP-628.

65. Xue J, Chen H, Xiong D, Huang G, Ai H, Liang Y, et al. Noninvasive Measurement of Glucose in Artificial Plasma with Near-Infrared and Raman Spectroscopy. Applied Spectroscopy, 2014;68(4):428-33.

66. Cássia R De, Borges F, Navarro RS, Giana HE, Tavares FG, Fernandes AB, et al. Detecting alterations of glucose and lipid components in human serum by nearinfrared Raman spectroscopy. Res Biomed Eng. 2015;31(2):160-8.

67. Berger AJ, Koo T, Itzkan I, Horowitz G, Feld MS. Multicomponent blood analysis by near-infrared Raman spectroscopy. Appl Opt. 1999;38(13):2916-26

68. Berger AJ, Itzkan I, Feld MS. Feasibility of measuring blood glucose concentration by near-infrared Raman spectroscopy. Spectrochim Acta A Mol Biomol Spectrosc. 1997;53:287-92.

69. Imai K. Clinical Chemistry and Immunoassay Testing Supporting the Individual Healthy Life. Hitachi Review. 2008;57:1-7.

70. Pas S, Molenkamp R, Schinkel J, Rebers S, Copra C, Seven-Deniz S, et al. Performance evaluation of the new Roche cobas AmpliPrep/cobas TaqMan HCV test, version 2.0, for detection and quantification of hepatitis C virus RNA. J Clin Microbiol. 2013 Jan;51(1):238-42.

71. Gammeren AJ Van, Gool N Van, Groot MJM De, Christa M. Analytical performance evaluation of the Cobas 6000 analyzer - special emphasis on trueness verification. Clin Chem Lab Med. 2008;46(6):863-71.

72. Ahmed A, Alam JM, Ali H, Sultana I, Fazal S. Comparative precision analysis of Precision-controls on automated chemistry analyzers. International Journal of Innovative Science Engineering and Technology. 2015;2(8):1-13. 